\section{REGISTROS Y BIOBANCOS DE ENFERMEDADES RARAS. UNA OPORTUNIDAD PARA AVANZAR}

Óscar Zurriaga

FISABIO-Salud Pública. Área de Investigación en Enfermedades Raras (Valencia) / Centro de Investigación Biomédica en Red (CIBER) en Epidemiología y Salud Pública (CIBERESP) ORCID iD: https://orcid.org/0000-0001-7279-432X zurriaga_osc@gva.es

Jacobo Martínez

FISABIO-Salud Pública. Unidad de Plataformas y Servicios (Valencia) ORCID iD: https://orcid.org/0000-0002-7540-8866

martinezjac@gva.es

Virginia Corrochano

Centro de Investigación Biomédica en Red (CIBER). Área Temática de Enfermedades Raras. CIBERER Biobank ORCID iD: https://orcid.org/0000-0003-1892-8352 vcorrochano@ciberer.es

Clara Cavero-Carbonell FISABIO-Salud Pública. Área de Investigación en Enfermedades Raras (Valencia) ORCID iD: https://orcid.org/0000-0002-4858-6456 cavero_cla@gva.es

Cómo citar este artículo/Citation: Zurriaga, O., Martínez, J., Corrochano, V. y Cavero-Carbonell, C. (2018). Registros y biobancos de enfermedades raras. Una oportunidad para avanzar. Arbor, 194 (789): a469. https://doi.org/10.3989/ arbor.2018.789n3011

Recibido: 30 abril 2015. Aceptado: 13 mayo 2016.

RESUMEN: Los registros y los biobancos de enfermedades raras se han convertido durante los últimos años en instrumentos clave para la investigación de este tipo de enfermedades. En el presente artículo se hace una revisión del concepto de registro y de la utilidad de los mismos en las enfermedades raras, se muestra un panorama de los registros de enfermedades raras en el ámbito internacional y nacional y se exponen los retos y las oportunidades que presentan los registros. Se presentan asimismo los biobancos como estructuras creadas con el fin de gestionar muestras biológicas para su uso en investigación. Se abordan los aspectos éticos, legales y sociales relacionados con la actividad de los biobancos y los aspectos técnicos asociados con la armonización, estandarización y control de calidad de las muestras y de la información relacionada y se ofrece un panorama general de los biobancos, recalcando su importancia en el ámbito de las enfermedades raras y la necesidad de colaboración con los registros de enfermedades raras.

PALABRAS CLAVE: Registro; biobanco; enfermedad rara; armonización; interoperabilidad; biobanking.

\section{RARE DISEASE REGISTRIES AND BIOBANKS: A WAY FORWARD}

Copyright: (C) 2018 CSIC. Este es un artículo de acceso abierto distribuido bajo los términos de la licencia de uso y distribución Creative Commons Reconocimiento 4.0 Internacional (CC BY 4.0).

ABSTRACT: In recent years, rare disease (RD) registries and biobanks have arisen as key tools for research into this group of pathologies. This paper reviews the concept of registries and their value in the RD field, providing an overview of RD registries at a national and international level. Challenges and opportunities of the registries are also dealt with. Similarly, biobanks are presented as structures created to manage biological samples for their use in research. Ethical, legal and social issues related to the activity of biobanks and technical issues associated with harmonization, standardization and quality control of samples and related information are addressed. An overview of biobanks is offered, emphasizing their importance in the RD field and the need for collaboration with $\mathrm{RD}$ registries.

KEYWORDS: Registry; biobank; rare disease; harmonization; interoperability; biobanking. 


\section{REGISTROS EN EL ÁMBITO DE LAS ENFERMEDADES RARAS}

\subsection{Introducción}

Disponer de información sistematizada es una necesidad generalizada en todas las disciplinas científicas. En el ámbito de las enfermedades raras (ER), los registros constituyen instrumentos clave para poder avanzar en la investigación clínica, en la mejora de la atención, en la vigilancia y en la calidad de vida de los afectados por una ER (Rare Diseases Task Force. Patient registries in the field of rare diseases).

\subsection{El concepto de registro}

En el ámbito de la salud suele distinguirse entre registro de pacientes y registro de enfermedades, aunque este es un asunto que va más allá de la diferencia semántica (Bellgard et al., 2013; Cavero-Carbonell et al., 2015).

Según el Diccionario de epidemiología (Porta, 2014), un registro de enfermedades es "el archivo o fichero de datos correspondientes a todos los casos de una enfermedad particular $u$ otra condición relevante de salud en una población definida de manera tal que pueda ser relacionado con una población base".

La definición de registro de pacientes que parece ser más aceptada es la proporcionada por la Agency of Health Research and Quality (Gliklich, Dreyer y Leavy, 2014) estadounidense: "sistema organizado que usa métodos de estudio de epidemiología observacional para recoger datos uniformes (clínicos y otros) con el fin de evaluar resultados específicos para una población definida por una enfermedad, condición o exposición particular, y que sirve a uno o más propósitos predeterminados, científicos, clínicos o de políticas".

Es habitual distinguir entre registros poblacionales o de población, que se refieren a poblaciones geográfica o administrativamente definidas y se proponen recoger todos los casos en esa población, y los no poblacionales, donde la cobertura no es completa o exhaustiva de toda la población y que están basados en centros sanitarios (hospitalarios u otros) o en otros criterios (asociaciones de pacientes, por ejemplo).

\subsection{La utilidad de los registros en el ámbito de las ER}

Lo que diferencia un tipo de registro de otro, sobre todo, es la finalidad: los registros de enfermedades poblacionales se dirigen principalmente a la vigilancia de la salud, mientras que los registros de pacientes están más orientados a la evaluación de resultados.
Los propósitos de salud pública e investigación figuran entre las principales utilidades de los registros en el ámbito de las ER, resaltadas en las recomendaciones principales del comité de expertos en ER europeo (EUCERD) sobre registros de pacientes y recogida de datos sobre ER (European Union Committee of Experts on Rare Diseases. EUCERD Core Recommendations on Rare Disease Patient Registration and Data Collection), que además considera las siguientes utilidades de los registros:

- Apoyar el desarrollo de políticas en el ámbito local, regional, nacional e internacional.

- Facilitar la investigación de las ER tanto en el campo clínico como en el epidemiológico.

- Monitorizar la provisión de atención sanitaria y de intervenciones terapéuticas.

- Facilitar la realización de ensayos clínicos y de estudios post-comercialización.

\subsection{La situación actual de los registros de ER}

\section{En el ámbito internacional}

Un hito importante ha sido la creación del consorcio IRDiRC (International Rare Diseases Research Consortium), cuyo objetivo para el año 2020 es desarrollar 200 nuevas terapias y diagnósticos para las ER. Entre las medidas y políticas que este consorcio propone (International Rare Diseases Research Consortium. Policies \& Guidelines) está "coordinar y conectar los registros de pacientes: establecer procedimientos operativos comunes estándar, armonizar las cuestiones éticas y de acceso a los datos y muestras de los pacientes", así como estimular a promover la armonización, interoperabilidad y acceso abierto a las ontologías que hay que aplicar a bases de datos, registros y biobancos. Los países miembros del consorcio han impulsado un importante número de proyectos, y en España uno de ellos ha sido el desarrollo de la red española de registros de ER para la investigación (Spanish Rare Diseases Registries Research Network, SpainRDR: https://spainrdr.isciii.es).

En Europa, las ER son un campo con un potencial de colaboración enorme (Montserrat Moliner y Waligora, 2013) y los registros no son ajenos a ello. La Unión Europea (UE) está apoyando los registros a través de la Plataforma europea para el registro de las ER (JRC Scientific and Technical Reports. Biobanks in Europe: Prospects for Harmonisation and Networking). Esta plataforma, establecida en el Joint Research Centre (JRC), proporciona servicios y he- 
rramientas comunes para los registros de ER de la UE. Y ya está desarrollando sus actuaciones en dos importantes redes de registros: EUROCAT (Vigilancia Europea de Anomalías Congénitas) y SCPE (Vigilancia de la Parálisis Cerebral en Europa). En los países de la UE, como cita la propia Comisión Europea (http://ec.europa.eu/health/rare_diseases/policy/ registries/index_en.htm), hay dos registros nacionales que son destacados: el Registro Nazionale Malatie Rare italiano (Taruscio, Kodra, Ferrari y Vittozzi, 2014) y el ya citado SpainRDR español.

La UE también ha financiado o cofinanciado con los estados miembros importantes proyectos o acciones conjuntas (AC) dedicados de manera directa o indirecta a los registros de ER. Entre ellos pueden citarse: el proyecto EPIRARE (European Platform for Rare Disease Registries: http://www.epirare.eu), la AC PARENT (cross-border PAtient REgistries iNiTiative: http://patientregistries.eu), la AC RD-ACTION (Data and policies for Rare Diseases: http://www.rd-action. eu) y también el proyecto RD-CONNECT (Integrated platform connecting databases, registries, biobanks and clinical bioinformatics for rare diseases research: http://rd-connect.eu).

El Comité de Expertos en ER de la Unión Europea (EUCERD), establecido en 2009 (Aymé y Rodwell, 2014) y transformado en 2014 en Grupo de Expertos de la Comisión Europea sobre ER, elaboró el ya citado conjunto de recomendaciones sobre registros de pacientes de ER y recogida de datos (EUCERD Core Recommendations on Rare Disease Patient Registration and Data Collection). Además, elaboró un informe (2014 Report on de State of the Art of Rare Disease Activities in Europe) en el que se repasaba la situación de los registros europeos de ER, detallando que, de acuerdo con la base de datos de Orphanet (Orphanet Report Series. Rare Disease collection. Rare Disease Registries in Europe), había 641 registros de ER en Europa (40 europeos, 74 globales, 446 nacionales, 77 regionales y 4 no definidos).

Precisamente Orphanet (http://www.orpha. net), el portal de información de referencia en ER y medicamentos huérfanos dirigido a una audiencia amplia, es un actor clave, ya que mantiene un listado de registros de ER, es la organización responsable de la gestión y mantenimiento de los números ORPHA, identificativos únicos asignados por Orphanet a una ER dada, y también es responsable de ORDO (Orphanet Rare Diseases Ontology: http://www.orphadata.org/cgi-bin/inc/ordo_ orphanet.inc.php), un vocabulario estructurado para las ER que expresa relaciones entre enfermedades, genes y otras características relevantes útiles para el análisis de las ER.

En los Estados Unidos de América, la Oficina de Investigación de Enfermedades Raras (ORDR) de los Institutos Nacionales de la Salud patrocinó un taller, tras el cual se estructuró un proyecto piloto conocido como Global Rare Diseases Patient Registry Data Repository/GRDR. Se trata de construir una infraestructura para un registro global, basado en internet, enlazado con bio-repositorios, que agregue información codificada y datos clínicos de pacientes de manera que este registro sirva tanto a pacientes como a investigadores y compañías farmacéuticas (Rubinstein et al., 2010).

\section{En el ámbito nacional}

En España, la experiencia llevada a cabo dentro de la Red Registros SpainRDR tuvo como objetivo la constitución de un Registro Nacional de ER en nuestro país a partir de los registros de pacientes orientados a la investigación de resultados y los registros de base poblacional dirigidos a la investigación epidemiológica, socio-sanitaria y a la planificación en salud. En este proyecto participaron las administraciones sanitarias de todas las comunidades autónomas españolas, el Ministerio de Sanidad, Servicios Sociales e Igualdad, el Centro de Referencia Estatal de Atención a Personas con Enfermedades Raras y sus Familias, seis sociedades médicas españolas, cuatro redes de investigación, organizaciones farmacéuticas y biotecnológicas, la Federación Española de Enfermedades Raras (FEDER) y su fundación, y el Instituto de Investigación de Enfermedades Raras (IIER), que actuó como coordinador de la red. Esta experiencia desembocó en la creación del Registro Estatal de Enfermedades Raras (REER) creado en 2015.

En España, como han mostrado los inventarios de registros realizados (López-Briones, Amorós, Benkovic y Zurriaga, 2014; Zurriaga Lloréns et al., 2006), existen diferentes registros de ER, con mayor o menor especificidad, en variados campos, pero es necesario destacar el ámbito de las anomalías congénitas. Así, de los 33 registros europeos miembros de pleno derecho de EUROCAT, dos de ellos son españoles (País Vasco y Comunidad Valenciana), y existe además un registro español afiliado (Navarra) y un miembro asociado español: el Estudio Colaborativo Español de Malformaciones Congénitas (ECEMC) (Martínez-Frías, 1995), establecido en 1976. 


\subsection{Los retos de los registros de ER y las oportunidades existentes}

Uno de los problemas que plantea la investigación en las $E R$ es que, a causa de su escasa frecuencia, una única organización no dispone de información del suficiente número de pacientes para realizar investigación clínica o traslacional que pueda ser generalizada. Esta es también una importante limitación que encuentran los registros de ER. Pero el principal reto es que, aunque algunas ER están cubiertas por más de un registro, muchas de ellas no están presentes en ninguno. Ello es debido a una conjunción de factores entre los que están:

- La escasa frecuencia de las ER, especialmente de las ultra-raras.

- La complejidad de desarrollar y gestionar un registro, que aumenta por el hecho de que los casos sean pocos.

- El desconocimiento y la falta de visibilidad de algunas ER.

Tampoco se puede dejar de mencionar la inespecificidad de los sistemas de clasificación y codificación: muchas de las ER no disponen de un código específico en la Clasificación Internacional de Enfermedades (CIE) o en otras clasificaciones de amplio uso (Schieppati, Henter, Daina y Aperia, 2008). Por ello es necesario que los sistemas sanitarios de información vayan confluyendo hacia sistemas mucho más específicos, como puede ser ORDO, o bien que se enlace con lenguajes con mayor especificidad (Rath et al., 2012) y que son internacionalmente reconocidos, como SNOMED-CT (Lee, Keizer, Lau y Cornet, 2014).

En el trabajo efectuado por RD-Connect (Thompson et al., 2014) se han sistematizado los retos a los que se enfrentan los registros de $E R$, como son:

- La continua reinvención de lo que es un registro.

- La fragmentación de los datos de los pacientes.

- Las dificultades de inclusión y seguimiento de los pacientes.

- La resistencia para adoptar y adaptarse a los elementos de datos comunes.

- Las dificultades para proteger la identidad del paciente, especialmente en los casos de enfermedades ultra-raras.

- La implementación de un identificador único de paciente, especialmente en lo que se refiere a los aspectos éticos de su utilización y a la protección de datos.
- La disponibilidad de las organizaciones e investigadores participantes para compartir datos.

Otros retos presentes también son:

- La complejidad de abordar un registro global que abarque un gran número de patologías y territorios. La tendencia recomendada por IRDiRC es que los registros no se restrinjan y no se dediquen a un único producto o intervención terapéutica.

- $\quad$ El origen de los datos: en muchas ocasiones las fuentes son básicamente hospitalarias, pero cada vez más se impone la aportación de otras fuentes como las de atención primaria (Phillips, 2004), así como las socio-sanitarias. La ampliación de fuentes es fundamental para evitar caer en sesgos (Dreyer y Garner, 2009).

- Alcanzar la interoperabilidad en los registros, en sus tres aspectos: técnico, semántico y organizacional.

- El aspecto transfronterizo, muy agudizado en el ámbito de la UE, que es necesario que ya sea abordado en los registros.

EUCERD (Aymé y Rodwell, 2014) sistematizó los retos más importantes para los registros de ER al citar el consenso establecido en torno a seis áreas principales, que son: la interoperabilidad internacional, las fuentes de datos, la recogida de datos, las buenas prácticas, el uso de datos para propósitos normativos y la sostenibilidad.

Además, las organizaciones de pacientes están demandando que sea tenido en cuenta el punto de vista de los pacientes y su participación en los registros. Y así, tres organizaciones internacionales, EURORDIS (European Organisation for Rare Diseases) en Europa, NORD (National Organization for Rare Disorders) en Estados Unidos y CORD (Canadian Organization for Rare Disorders) en Canadá, efectuaron una declaración conjunta (EURORDIS-NORD-CORD Joint Declaration 10 Key Principles of Rare Disease Patient Registries) que recoge los diez principios clave para los registros de pacientes. Entre ellos citan aspectos generales de los registros, que ya han sido mencionados, y otros más específicos concernientes a los pacientes, como son la inclusión de datos directamente suministrados por los pacientes, acompañados de datos notificados por profesionales sanitarios; el refuerzo de la cooperación público-privada para asegurar la sostenibilidad de los registros; la implicación de los pacientes en la gobernanza y gestión de los registros; o el papel que podrían jugar los 
registros de pacientes para la construcción y el empoderamiento de comunidades de pacientes.

Todos estos retos, afortunadamente, se presentan en un momento en el que las ER han entrado en la agenda de muchos países e instituciones supranacionales, de manera que aspectos como la colaboración internacional, las oportunidades de financiación, la existencia de planes o estrategias nacionales de ER, el impulso a la investigación, el desarrollo de plataformas (nacionales o internacionales) y otros favorecen el desarrollo y consolidación de los registros de ER.

El trabajo desarrollado por diferentes proyectos permite disponer ya de aspectos resueltos como, por ejemplo, los elementos de datos comunes para los registros de ER que han sido estudiados y sistematizados en EpiRare (Common Data Set and disease-, treatment and other specific modules).

Las oportunidades de desarrollo y estabilización de los registros afortunadamente existen.

\section{BIOBANCOS EN EL ÁMBITO DE LAS ER}

\subsection{Introducción}

Según la definición recogida en la Ley de Investigación Biomédica (LIB 14/2007) un biobanco es "un establecimiento público o privado, sin ánimo de lucro, que acoge una colección de muestras biológicas concebida con fines diagnósticos o de investigación biomédica y organizada como una unidad técnica con criterios de calidad, orden y destino".

En la práctica, un biobanco es una plataforma de apoyo a la investigación que actúa de nexo de unión entre donantes, clínicos e investigadores con el propósito de asegurar un tratamiento seguro y eficaz de las muestras biológicas y datos asociados. Los biobancos son repositorios de muestras biológicas almacenadas con fines de investigación médico-científica que son recogidas bien con esos fines o que constituyen muestras residuales de procedimientos médico-quirúrgicos, y su valor depende no solo de la muestra en sí sino de la información clínica asociada de que se disponga. Existen diferentes tipos de biobancos que, por lo general, se clasifican en biobancos poblacionales, biobancos orientados a enfermedades, biobancos de casos-control o bancos de tejidos, entre otros (Riegman, Dinjens y Oosterhuis, 2007; Riegman, Morente, Betsou, Blasio y Geary, 2008).

Estos repositorios constituyen estructuras bien organizadas en el seno de instituciones de distinto carácter, tales como hospitales o centros de investi- gación, fundamentalmente. Precisan para su funcionamiento en nuestro país de una autorización otorgada por la autoridad sanitaria competente en cada caso, así como de su inclusión en el Registro Nacional de Biobancos del Instituto de Salud Carlos III (Real Decreto 1716/2011).

\subsection{Justificación}

Las muestras biológicas han desempeñado durante décadas un papel esencial en el desarrollo de la investigación académica (Artene et al., 2013). Hasta hace pocos años, la investigación médica estaba basada en la disponibilidad de muestras por parte de individuos o pequeños grupos de investigación. En los últimos años, los avances en investigación genómica y proteómica han permitido diferenciar enfermedades multifactoriales, previamente diagnosticadas como un único desorden, en entidades diagnósticas diferentes, abriendo el campo a la medicina personalizada (Botti, Franco, Cantile, Ciliberto y Ascierto, 2012). Para ello, es imprescindible poder contar con una amplia colección de muestras biológicas y sus datos asociados, tanto de individuos afectados como de individuos sanos que constituirán grupos de control. Todo ello ha motivado el surgimiento y el desarrollo de repositorios de muestras biológicas que necesariamente habrán de colaborar y armonizar sus prácticas con el resto de los repositorios. El proceso de armonización, así como el de regulación por parte de las autoridades competentes, ha contribuido a que los biobancos se constituyan en garantes de la seguridad y de la calidad de las muestras biológicas almacenadas, lo que redunda a su vez en la calidad de los resultados de investigación.

En el caso particular de las ER, donde el número de afectados es tan bajo y los casos se encuentran tan dispersos, la figura del biobanco cobra aun mayor relevancia.

A continuación, se comentan los aspectos más relevantes relacionados con la gestión de los biobancos.

\subsection{Aspectos legales, éticos y sociales}

"Las actividades desempeñadas por los repositorios de muestras biológicas conllevan muchos dilemas éticos y legales, por lo que tanto en el ámbito nacional como internacional existen diversas normas establecidas para salvaguardar los derechos de los donantes" (Veloza Cabrera, Wiesner Ceballos, Serrano López, Peñaranda Correa y Huertas Salgado, 2010). En el caso concreto de España, hay regulaciones de carácter general y específico que son de aplicación. Entre ellas, 
la Ley de Investigación Biomédica 14/2007, la Ley Orgánica de Protección de Datos, LOPD, 15/1999, y el Real Decreto 1716/2011. Estos documentos resaltan el hecho de que los intereses de los seres humanos prevalecen sobre los intereses de la sociedad y de la ciencia (Medical Research Council. Human tissue and biological samples for use in research. Operational and ethical guidelines) y regulan las distintas actividades que se llevan a cabo en estos establecimientos.

En cuanto a las diferentes consideraciones éticas y legales hay que tener en cuenta las siguientes:

\section{a. Procedencia de las muestras}

La procedencia condiciona el posible uso: en el caso de las muestras obtenidas en el curso de intervenciones clínicas, estas solo se pueden almacenar en un biobanco cuando existe un excedente; en ningún caso se pueden ver afectados los estudios diagnósticos. En cuanto a las muestras obtenidas para investigación, su uso se encuentra limitado a un proyecto de investigación en concreto a no ser que el individuo consienta otros usos.

\section{b. Protección de los riesgos de la investigación}

Los beneficios de participar en los biobancos no son individuales sino colectivos y están representados por todos los avances en los conocimientos científicos para la prevención, el tratamiento y el diagnóstico de las enfermedades. La naturaleza y el grado de los riesgos derivados de la actividad científica de los biobancos depende fundamentalmente de la capacidad de identificación de las muestras y de los datos asociados a ellas.

Los riesgos derivados de la investigación pueden ser intrínsecos o ulteriores. Entre los primeros se incluyen los de carácter biológico o psicosocial. Los primeros están representados por las complicaciones derivadas de la toma de la muestra y los riesgos psicosociales por la violación de la confidencialidad y de la privacidad. En cuanto a los riesgos ulteriores, las muestras biológicas contenidas en los biobancos tienen el potencial de generar una cantidad de datos en el futuro que también deben estar sujetos a regulaciones sobre la protección de datos personales y sobre la confidencialidad para evitar el riesgo de discriminación genética.

Con el fin de obtener la autorización por parte del comité de ética correspondiente, se deberá demostrar que los riesgos y los beneficios están equilibrados y que los posibles riesgos han sido minimizados.

c. Documento de consentimiento informado

Toda muestra ha de ir obligatoriamente acompañada de un consentimiento informado, definido como "un acuerdo por escrito mediante el cual el sujeto de investigación o, en su caso, su representante legal autoriza su participación en la investigación con pleno conocimiento de la naturaleza de los procedimientos, beneficios y riesgos a que se someterá, con la capacidad de libre elección y sin coacción alguna" (LIB 14/2007).

En el caso de los biobancos, por lo general, la naturaleza y el rol del consentimiento informado varían en relación con el consentimiento tradicional, en el cual el sujeto autoriza el uso de sus muestras en determinados proyectos de investigación. Así, la tendencia actual es solicitar al donante la firma de un consentimiento informado de carácter más amplio que permita el uso de las muestras en distintos proyectos, si bien el donante puede revocar el consentimiento en cualquier momento.

d. Acceso a las muestras y datos por parte de los investigadores

Los biobancos deben tener políticas bien establecidas para distribuir las muestras a los investigadores, así como procedimientos estandarizados para determinar lo que constituye un uso apropiado en investigación de las muestras y datos (European Commission. Biobanks for Europe. A challenge for governance. Report of the Expert Group on Dealing with Ethical and Regulatory Challenges of International Biobank Research). La solicitud de muestras debe estar, por tanto, bajo un estricto nivel de revisión científica por parte de un comité científico. La cesión de muestras debe además quedar aprobada por el comité de ética al que el biobanco en cuestión esté adscrito.

\subsection{Estandarización, normalización y control de calidad}

La capacidad de los biobancos de poner a disposición de los investigadores muestras e información asociada de la más alta calidad requiere de la implantación en su organización de buenas prácticas (BP) de laboratorio, del desarrollo de procedimientos normalizados de trabajo (PNT) y del tratamiento informático de los datos asociados, así como de la implantación de un sistema que garantice la calidad (Betsou et al., 2007; Liaño y Torres, 2009; Morente, Fernández y Álava, 2008).

Dada la importancia que presenta la colaboración internacional en la investigación biomédica, la armonización de las directrices sobre las BP, la estandarización y normalización de los procedimientos en los biobancos y el despliegue de políticas de aseguramiento de la calidad son una prioridad (Red Nacional de Biobancos (RNB). Plan estratégico de la RNB). 
Diversas organizaciones internacionales han desarrollado durante los últimos años guías de BP en las que se recogen políticas y directrices para las mejores prácticas, pudiendo destacar, entre otras, las desarroIladas por la Internacional Society for Biological and Environmental Repositories (Astrin, Baker y Barr, 2012), la Organization for Economic Co-operation and Development (OECD Best Practice Guidelines for Biological Resource (entres), la Office of Biorepositories and Biospecimen Research, la International Agency for Research on Cancer o la Australasian Biospecimen Network.

Los procedimientos de trabajo en los biobancos deben basarse en las directrices establecidas en este tipo de guías. De igual modo, la estandarización de los procedimientos debe basarse en la evidencia científica, y debe evolucionar como resultado de los avances en la investigación.

La normalización de los procedimientos, por su parte, adquiere un papel fundamental ante una necesidad muy concreta: la conexión en red entre biobancos (Simeon-Dubach, Burt y Hall, 2012). Así, la creación de redes de biobancos, o la participación en proyectos multicéntricos requiere que todos los implicados adopten unos procedimientos de operación comunes (Norlin et al., 2012).

El trabajo en red entre biobancos precisa además de un flujo de información multidireccional y, en este sentido, una correcta gestión y trazabilidad de las variables pre-analíticas asociadas a las muestras resulta clave a la hora de garantizar que la interconexión y la interoperabilidad entre biobancos sea efectiva (Asslaber y Zatloukal, 2007). En el año 2010, el Working Group on Biospecimen Science de la International Society of Biological and Environmental Repositories (ISBER) publicó un artículo en el que se presentaba la primera versión de la codificación Simple PREanalytical Code (SPREC) (Betsou et al., 2010). Esta codificación pretende sintetizar las condiciones pre-analíticas asociadas al material biológico, desde su extracción hasta la utilización por el investigador, y ha supuesto una revolución en el ámbito de los biobancos (SimeonDubach y Watson, 2014).

Entrando ya en materia de aseguramiento de la calidad, los biobancos deben implantar un sistema de gestión de la calidad que consiga que la organización funcione de manera eficaz (Haro, Regalado y GarcíaMontero, 2006). Aunque en el año 2008 la Asociación Francesa de Normalización (AFNOR) desarrolló la primera norma de calidad específica de Biobancos (NFS96-9000), no existe una norma internacional es- pecífica para estos establecimientos y es la norma ISO 9001/2015 la que está siendo adoptada en España y en Europa. Sin embargo, considerando el desarrollo que han experimentado los biobancos durante los últimos años, se requieren normas adicionales en ensayos, calibración (ISO 17025/2005) y producción de material de referencia (Betsou et al., 2007).

\subsection{Situación en Europa}

En la última década en muchos países europeos se han establecido biobancos poblacionales $u$ orientados a enfermedades, y en ellos se ha hecho una fuerte inversión tanto científica como financiera (European Commission. Biobanks for Europe. A challenge for governance), habiendo recogido hasta la fecha millones de muestras que han favorecido grandes avances en la comprensión de factores de riesgo específicos de enfermedades complejas.

En 2010, el European Institute for Prospective Technological Studies (IPTS) del European Commission's Joint Research Centre, en colaboración con el European Science and Technology Observatory (ESTO), publicó los resultados del estudio Biobanks in Europe: Prospects for Harmonisation and Networking, que puso de manifiesto la variación y fragmentación de las prácticas y actividades de los biobancos dentro de Europa. Sobre la base de esta evidencia, el informe recomendaba la creación de una red internacional o de una organización "paraguas" que fomentase la armonización y la normalización de las prácticas de los distintos biobancos (European Commission. Biobanks for Europe. A challenge for governance).

Así, la constitución de redes y la armonización se han convertido en cuestiones clave en el ámbito de los biobancos y, a fin de garantizar una investigación en colaboración significativa y superar la heterogeneidad de los biobancos en Europa, se procedió a la elaboración de directrices comunes y PNT y a la armonización de metodologías.

Para dar respuesta a estas necesidades, han surgido importantes iniciativas. Entre ellas, Public Population Project in Genomics (BOX 4), PHOEBE, EuroBioBank, GenomeEUtwin, TuBa-Frost y NUGENOB. Además, la Organización para la Cooperación y el Desarrollo Económicos (OCDE) y el U. S. National Cancer Institute ( $\mathrm{NCl}$ ), así como la International Society of Biological and Environmental Repositories (ISBER), han establecido directrices y recomendaciones específicas.

Por último, la Comisión Europea, ha brindado apoyo a través de la financiación de proyectos colaborativos 
en el 7.o Programa Marco. Ejemplos de ello son la fase preparatoria del Biobanking and Biomolecular Resources Research Infrastructure (BBMRI) o los proyectos TISS.EU, BioSHARE-EU y RD-Connect, financiados por la Unión Europea en el marco del International Rare Diseases Research Consortium (IRDiRC).

Además de los asuntos relacionados con la armonización, la práctica de los biobancos se enfrenta a numerosos desafíos. Mientras las redes y consorcios van en aumento, quedan muchos otros puntos por solventar, tales como el flujo transfronterizo de muestras y datos asociados, la integración informática de distintos tipos de datos o la sostenibilidad a largo plazo de estas estructuras.

La Red de EuroBioBank es la primera y la única red operativa de biobancos en Europa que proporciona muestras humanas como un servicio a la comunidad científica para el desarrollo de la investigación en ER. Está compuesta por 24 miembros procedentes de 11 países europeos y sus aproximadamente 150.000 muestras están disponibles a través de un catálogo online. Esta red fue creada en 2001 por la Asociación Francesa contra la Miopatía (AFM) y EURORDIS con el fin de contribuir a acelerar la investigación en $E R$, siendo su principal objetivo desarrollar una masa crítica de muestras biológicas y colecciones para ER.

\subsection{Situación en España}

La investigación biomédica en España ha sufrido un importante desarrollo en las últimas tres décadas, convirtiendo a la comunidad investigadora española en la novena potencia en producción científica (Red Nacional de Biobancos (RNB). Plan estratégico de la $R N B$ ). La investigación biomédica española se encuentra totalmente equiparada a la de los países de nuestro entorno y en este sentido podemos afirmar que el desarrollo experimentado por los biobancos de nuestro país durante la última década se enmarca dentro de la tendencia de crecimiento de la actividad de biobanking en Europa.

En el año 2006, el Instituto Roche realizó una encuesta para detectar la situación real con la que se enfrentaba España respecto a la obtención de muestras para estudios biomédicos. A partir de esta encuesta se desarrolló una Guía práctica para la utilización de muestras biológicas en Investigación Biomédica, en la que se revisaron las condiciones de los biobancos en España y en la que se concluyó que, aunque los biobancos eran elementos cuya importancia para la investigación biomédica era ya reconocida, necesitaban un impulso aún mayor. Se hacía fundamental modernizar sus instalaciones, mejorando los controles de calidad y la preparación de sus técnicos, además de aumentar el tiempo de dedicación que prestan estos últimos a los bancos. Su colaboración con otros biobancos o su funcionamiento en red es capital para la expansión de estos establecimientos. Y todo ello requería un sistema de acreditación que definiera qué es y qué no es un biobanco, y que señalara las competencias de aquellos que realmente lo fueran frente a otros que no constituyeran más que meras colecciones.

En este contexto, la aprobación y entrada en vigor de la LIB 14/2007 marcó el proceso de reestructuración, desarrollo y consolidación que han experimentado los biobancos en nuestro país. La ley, que regulaba por primera vez la figura de los biobancos con fines de investigación biomédica, establece criterios y líneas de actuación en aspectos relacionados con su organización, funcionamiento, autorización, cierre, etc.

La existencia de un marco legal específico de biobancos resultó muy beneficiosa a la hora de abordar la problemática con la que se encontraba el sector. Sin embargo, la ley resultó incompleta para algunos aspectos relacionados con la incorporación de muestras a los biobancos, con el régimen de las colecciones de acuerdo con su finalidad (ensayos clínicos, líneas de investigación, diagnóstico...) o con la custodia de los consentimientos informados, entre otros.

Bajo el amparo de la LIB 14/2007, el Instituto de Salud Carlos III (ISCIII) puso en marcha en 2010 una iniciativa de creación de una estructura cooperativa en red (RE$\mathrm{TIC}$ ) de biobancos hospitalarios, denominada Red Nacional de Biobancos (RNBB), con el objetivo de sentar las bases para convertirse en una plataforma trasversal dentro del sistema nacional de I+D+i. La red contaba con el compromiso añadido de situar a nuestro país en la vanguardia de las iniciativas europeas a este respecto, formando parte del proyecto ESFRI de BBMRI.

La concesión de dichas ayudas determinó el modelo organizativo de los biobancos que en la actualidad nos encontramos en los hospitales del sistema nacional de salud, ya que estuvo condicionada a la creación de un único biobanco por centro que acogiera el conjunto de colecciones de muestras obtenidas en los distintos servicios del hospital. Se trata de un modelo multidisciplinar de biobanco, conocido con el nombre de biobanco hospitalario, que caracteriza, en gran medida, la situación de los biobancos en España.

La RNBB quedó integrada por un total de 52 centros hospitalarios y de 11 centros asociados con actividad relevante en biobancos, entre los cuales se encon- 
traban biobancos poblacionales y otras plataformas cooperativas de ámbito autonómico, de ámbito interterritorial y del Centro de Investigación Biomédica en Red (CIBER).

Entre los años 2010 y 2013, la RNBB promovió un espacio colaborativo con resultados notables en armonización de procedimientos, difusión al exterior, formación de profesionales, construcción de una primera fase de una plataforma informática de interoperabilidad técnica y semántica, promoción de criterios de planificación económica y de políticas de aseguramiento de la calidad, etc. (Red Nacional de Biobancos (RNB). Plan estratégico de la RNB).

En el periodo comprendido entre 2014 y 2017, la Plataforma Red Nacional de Biobancos (PRNBB) ha dado continuidad a la experiencia previa como RETIC, como Plataforma de Apoyo a la Investigación en Ciencias y Tecnologías de la Salud del Instituto de Salud Carlos III (ISCIII). En esta etapa la PRNBB, formada por 52 instituciones de 15 Comunidades Autónomas, ha afrontando nuevos retos, llevando a cabo una gestión más profesionalizada de sus servicios, la promoción de colecciones de alto valor estratégico y la realización de actividades de I+D+i en el campo de los biobancos.

En la actualidad España cuenta con un total de 94 biobancos inscritos en el Registro Nacional de Biobancos y la PRNBB se encuentra en fase de preparación de un Plan de Actuación para los años 2018-2021, que se enmarca en la Acción Estratégica en Salud del ISCIII. Realizando un análisis comparativo entre la situación actual de los biobancos españoles con respecto a la situación descrita en el año 2006 en la Guía Práctica para la Utilización de Muestras Biológicas en Investigación Biomédica, podemos afirmar que ha mejorado de una forma sustancial. La existencia de un marco legislativo específico para biobancos, la financiación proporcionada por el ISCIII y por todas las instituciones que han apostado por la consolidación o creación de biobancos, el trabajo realizado desde la PRNBB y desde otras iniciativas de redes de biobancos o biobancos en red y, por supuesto, el realizado por todos los profesionales involucrados en el sector han sido piezas clave en el proceso de saneamiento, estructuración y organización de los biobancos españoles.

\subsection{Biobancos en el ámbito de las ER}

Si bien el papel de los biobancos en el avance de la investigación es indiscutible, en el ámbito de las ER los biobancos adquieren aún mayor relevancia. A pesar de que los biobancos de ER comparten muchos aspectos con el resto de biobancos, la rareza y diversidad de las ER y sus muestras biológicas y datos asociados plantean retos y oportunidades específicas que requieren de la colaboración transnacional y la armonización. Así, pequeñas colecciones o incluso muestras individuales pueden ser extremadamente valiosas para la investigación, teniendo algunas de ellas relevancia directa para la salud de determinados pacientes. Es además en este campo donde el trabajo en colaboración con otros biobancos cobra mayor importancia, de manera que se llegue a cierto grado de armonización (incluyendo la codificación de ER) de forma que las muestras de distintos biobancos puedan ser integradas en estudios a mayor escala.

Existen en la actualidad más de 100 biobancos de ER en la Unión Europea (Lochmüller et al., 2009/2010). En nuestro país, destaca la iniciativa puesta en marcha en 2008 por el Centro de Investigación Biomédica en Red de Enfermedades Raras (CIBERER - Instituto de Salud Carlos III), el CIBERER Biobank (CBK), un biobanco de carácter público y sin ánimo de lucro ubicado en la Fundación para el Fomento de la Investigación Sanitaria y Biomédica de la Comunidad Valenciana (FI$\mathrm{SABIO}$ ) en Valencia.

La integración del CBK dentro de la estructura del CIBER, Área de Enfermedades Raras (CIBERER), establecida para servir de referencia, coordinar y potenciar la investigación de excelencia sobre las ER en España, confiere al biobanco ese mismo carácter, el de ser un centro donde se prima y favorece la colaboración con grupos de investigación básica y clínica, haciendo especial hincapié en los aspectos de la investigación genética, molecular, bioquímica y celular de las ER con el objetivo de mejorar el conocimiento sobre la epidemiología, las causas y los mecanismos de producción de las mismas.

Con esta iniciativa, el CIBERER ha querido dar respuesta a los miles de ER para las que no alcanza a desarrollar proyectos de investigación, proporcionando material biológico para que otros grupos de investigación puedan llevarlos a cabo. Asimismo, el CIBERER ha querido solventar uno de los problemas principales con que se encuentra la investigación en este tipo de patologías: la dispersión de muestras debida a la baja prevalencia de las mismas.

Una segunda iniciativa del ISCIII al respecto corresponde a la creación en 2013 del Biobanco Nacional de Enfermedades Raras (BioNER), conectado con el Registro Nacional de ER (SpainRDR). El BioNER se constituye sobre la base del Biobanco del Instituto de Investigación de Enfermedades Raras (IIER), bioban- 
co que alberga muestras biológicas de afectados por ER, trastornos del espectro autista y causados por el síndrome del aceite tóxico, y que participa en varias redes de biobancos y proyectos nacionales como internacionales, tales como la RetBIOH, Eurobiobank o RD-Connect.

\subsection{Perspectivas de futuro}

Aunque la actividad de coleccionar muestras biológicas para su utilización en investigación constituye un hecho histórico en medicina (Morente et al., 2008), el biobanking puede considerarse como una disciplina nueva y muy dinámica que surge ante la necesidad de disponer de material biológico e información asociada de calidad para su puesta a disposición de la comunidad científica internacional (Hewitt, 2011).

Como ya hemos visto en puntos anteriores, los biobancos en España y en los países de nuestro entorno han experimentado un enorme desarrollo durante los últimos años (Morente, Álava y Fernández, 2007). En términos generales podemos afirmar que este desarrollo ha venido acompañado de una evolución en la forma de entender el mundo del biobanking. En una primera etapa, los biobancos centraron sus prioridades en el número de muestras y en la cantidad de información que debía asociarse a las mismas. Durante una segunda etapa, en la que nos encontramos actualmente, el biobanking está centrando sus prioridades en la calidad de las muestras y de la información asociada. Ambas etapas han estado muy orientadas al producto final y a los desarrollos internos operacionales de los propios biobancos (Simeon-Dubach y Watson, 2014).

En nuestros días se está proponiendo que los biobancos evolucionen hacia una nueva etapa en la que se priorice el valor y el impacto de su actividad, en la que los donantes sean conscientes del beneficio del uso de sus muestras en investigación biomédica, en la que las entidades financiadoras valoren los resultados en términos de mejora de la investigación y de producción científica etc., y en la que los investigadores aprovechen la capacidad de plasmar sus ideas en generación de conocimiento. La nueva forma de entender los biobancos se fundamentaría en su sostenibilidad desde una triple perspectiva: social, financiera y operativa. En este sentido se plantea la necesidad de reenfocar las actividades, servicios y productos de los biobancos priorizando las necesidades de donantes, financiadores y clientes (Simeon-Dubach y Watson, 2014).

Por otra parte, la experiencia de los últimos años nos está permitiendo confirmar el hecho de que las muestras que se obtengan hoy van a ser las utilizadas en la investigación del mañana. En este sentido, tendremos que hacer el esfuerzo de prever el tipo de investigación que se llevará a cabo a medio plazo. La revolución de las denominadas ómicas, junto con los avances en investigación en células madre etc., han estado asociados al desarrollo de tecnologías que evolucionan de una forma imparable y que hacen, a su vez, que evolucione también el tipo de muestras que estas demandan para su uso en investigación (Kreiner e Irion, 2013). Así, de cara a los próximos años, se hace necesario el desarrollo de biobancos en los que, por poner un ejemplo, se almacenen nuevos tipos celulares con perfiles genéticos conocidos que faciliten el desarrollo de nuevas terapias.

Estos aspectos adquieren un papel muy importante en el caso de los biobancos de ER por las particularidades que estos presentan.

\section{REGISTROS Y BIOBANCOS: UNA UNIÓN NECESARIA}

En casi todas las recomendaciones citadas, de diferentes organismos e instituciones, se menciona que los datos de los registros de pacientes de ER deben estar enlazados con los correspondientes datos de los biobancos.

Así, el consorcio IRDiRC expone que la interoperabilidad y la armonización entre registros y biobancos debería ser un objetivo perseguido constantemente. $Y$ que el enlace y la transferencia de datos entre las plataformas existentes debería ser considerada como una de las "buenas prácticas" entre registros y biobancos.

Este enlace debe permitir compartir y enriquecer mutuamente la información disponible sobre los pacientes con ER, posibilitando avanzar en la investigación y mejora diagnóstica y terapéutica. Pero requiere que se respeten escrupulosamente las consideraciones éticas, las medidas de control de calidad y protección de datos y necesita además un gran avance en la interoperabilidad. Registros y biobancos han vivido, en muchas ocasiones, dándose la espalda, pero afortunadamente ya se dan las condiciones para solventar los aspectos técnicos, semánticos y de organización que permitan superar esta situación, de manera que los datos de los registros de pacientes de ER estén enlazados con las muestras biológicas de los biobancos, permitiendo mayores avances en la investigación.

\section{CONCLUSIONES}

Los registros son reconocidos como una prioridad global en el campo de las ER, pero existe todavía un amplio y largo camino por desarrollar. 
La colaboración internacional y el respaldo que las instituciones y organizaciones están dado al desarrollo de los registros ha permitido, en muchos casos, su puesta en marcha. Ahora es necesario asegurar además su sostenibilidad. Los registros son instrumentos que requieren tiempo para alcanzar su máxima potencialidad y que, por desgracia, tardan un poco en ofrecer datos con la calidad requerida. Hay que ser paciente, dentro de la exigencia.

Los aspectos técnicos necesarios para el desarrollo de un registro son conocidos, aunque en muchas ocasiones se actúa como si no lo fueran y se producen reinventos. En cambio, los aspectos semánticos actúan, en el campo de las ER, como un freno importante de los registros. Es necesario seguir avanzando en la confluencia hacia sistemas que sean apropiados y den respuestas adecuadas y en los que la especificidad y multiplicidad de enfermedades estén contempladas.

La gestión, gobernanza y organización de los registros y su conexión con otras plataformas, como los biobancos, son aspectos mejorables y en los que se debe seguir haciendo hincapié. También deben contemplarse las demandas de participación de los pacientes.

El propósito y justificación de los registros está claramente establecido, pero es un aspecto que no hay que perder nunca de vista, dado que los datos de los pacientes concitan muchos intereses, legítimos en la mayoría de los casos, pero en otras ocasiones alejados del beneficio directo de estos.

El concepto de biobanco como repositorio que recoge, almacena y distribuye material biológico y su información clínica asociada emerge como estrategia de apoyo a la investigación clínica y traslacional, constituyendo un recurso esencial para el diagnóstico, la investigación basada en la genómica, proteómica y metabolómica, la investigación terapéutica y la búsqueda de biomarcadores, entre otros.

Por ello, no es de extrañar el enorme desarrollo que han experimentado en los últimos años en materia de armonización, normalización, creación de redes, control de la calidad, etc., si bien quedan importantes cuestiones de carácter ético, de sostenibilidad económica, de fomento de la cooperación con la industria etc. que están por resolver.

En este sentido, las iniciativas actuales de estandarización y armonización como SPREC o la implantación sistemas de gestión de la calidad con PNTs comunes, garantizan un óptimo aprovechamiento de los recursos disponibles con el propósito de satisfacer la necesidad de comparación de las muestras y la información clínica asociada a las donaciones de material biológico humano. Estos elementos son clave en la próxima generación de biobancos y serán fundamentales para el desarrollo de una plataforma europea potente en materia de investigación biomédica. Todo lo anterior, sin olvidar que el fin último de los biobancos es lograr que los beneficios resultantes de las investigaciones que empleen muestras biológicas humanas sean compartidos con la sociedad en general y con la comunidad internacional. Estos beneficios necesariamente han de redundar en la mejora de la atención a los afectados por alguna enfermedad, en la mejora de los servicios de salud o en el refuerzo de las capacidades de investigación.

Los retos a los que se enfrentan los registros y los biobancos en el campo de las ER son muchos, pero las oportunidades existen y deben ser aprovechadas. Lo que se puede obtener es mucho y los pacientes y sus allegados lo necesitan.

\section{BIBLIOGRAFÍA}

Artene, S. A., Ciurea, M. E., Purcaru, S. O., Tache, D. E., Tataranu, L. G., Lupu, M. y Dricu, A. (2013). Biobanking in a Constantly Developing Medical World. The Scientific World Journal, 2013, 343275. https://doi. org/10.1155/2013/343275

Asslaber, M. y Zatloukal, K. (2007). Biobanks: transnational, European and global networks. Briefings in Functional Genomics and Proteomics, 6 (3), pp.193-201. https://doi. org/10.1093/bfgp/elm023
Astrin, J., Baker, S. y Barr, T. J. (2012). Best practices for repositories: collection, storage, retrival, and distribution of biological materiasl for research. Biopreserv Biobank, 10 (2), pp. 79-161.

Aymé, S. y Rodwell, C. (2014). The European Union Committee of Experts on Rare Diseases: three productive years at the service of the rare disease community. Orphanet Journal of Rare Diseases, 9, pp. 30-36. https://doi.org/10.1186/1750-1172-9-30

Bellgard, M., Beroud, C., Parkinson, K., Harris, T., Ayme, S., Baynam, G. [...] y
Hunter, A. (2013). Dispelling myths about rare disease registry system development. Source Code for Biology and Medicine, 8 (1), pp. 21-28. https:// doi.org/10.1186/1751-0473-8-21

Betsou, F., Luzergues, A., Carter, A., Geary, P., Riegman, P., Clark B. [...] $y$ the Marble Arch Working Group on International Biobank (2007). Towards norms for accreditation of biobanks for human health and medical research: Compliation of existing guidelines into an ISO certification/accreditation norm-com- 
patible format. The Quality Assurance Journal: The Quality Assurance Journal for Pharmaceutical, Health and Environmental Professionals, 11 (3-4), pp. 221-294. https://doi. org/10.1002/qaj.425

Betsou, F., Lehman, S., Ashton, G., Barnes, M., Benson, E. E., Coppola, D. [...] y Gunter, E. (2010). Standard preanalytical coding for biospecimens: defining the sample PREanalytical code. International Society for Biological and Environmental Repositories (ISBER), 19 (4), pp. 1004-1011. https://doi.org/10.1158/1055-9965. EPI-09-1268

Botti, G., Franco, R., Cantile, M., Ciliberto, G. y Ascierto P. A. (2012). Tumor biobanks in translational medicine. Journal of Translational Medicine, 10, 204. https://doi.org/10.1186/14795876-10-204

Caboux, E., Plymoth, A. y Hainaut, P. (eds.), (2007). International Network of Biological Resource Centres for Cancer Research: Recommendations on Common Minimal Technical Standards. World Health Organization Press.

Cavero-Carbonell, C., Gras-Colomer, E., Guaita-Calatrava, R., López-Briones, C., Amorós, R., Abaitua, I. [...] y Zurriaga, O. (2015). Consensus on the criteria needed for creating a raredisease patient registry. A Delphi study. Journal of Public Health, 38 (2), e178-e186. https://doi.org/10.1093/ pubmed/fdv099

Dreyer, N. A. y Garner, S. (2009). Registries for robust evidence. JAMA, 302 (7), pp. 790-791. https://doi. org/10.1001/jama.2009.1092

Gliklich R., Dreyer N., Leavy M. (eds.). (2014). Registries for Evaluating Patient Outcomes: A User's Guide (3 ${ }^{\text {rd }}$ ed.). AHRQ Publication No. 13 (14)EHC111. Rockville, MD: Agency for Healthcare Research and Quality.

Haro, M. J., Regalado, A. M., García-Montero, A. C. (2006). Gestión de calidad en biobancos. En: Guía práctica de utilización de muestras biológicas en investigación biomédica. Instituto Roche, pp. 103-119.

Hewitt, R. E. (2011). Biobanking: the foundation of personalized medicine. $\mathrm{Cu}$ rrent Opinion in Oncology, 23 (1), pp. 112-119. https://doi.org/10.1097/ cC0.0b013e32834161b8
Kreiner, T. e Irion, S. (2013). Wholegenome analysis, stem cell research, and the future of biobanks. Cell Stem Cell, 12 (5), pp. 513-516. https://doi. org/10.1016/j.stem.2013.04.024

Lee, D., Keizer N. de, Lau, F. y Cornet, R. (2014). Literature review of SNOMED CT use. Journal of the American Medical Informatics Association, 21 (e1), pp. e11-e19. https://doi. org/10.1136/amiajnl-2013-001636

Liaño, F. y Torres, A. M. (2009). Biobancos: una nueva herramienta para la investigación clínica. Nefrología, 29 (3), pp. 193-195.

Lochmüller, H., Aymé, S., Pampinella, F., Melegh, B., Kuhn, K. A., Antonarakis, S. E. y Meitinger, T. (2009/2010). The Role of Biobanking in Rare Diseases: European Consensus Expert Group Report. Biopreservation \& Biobanking, 7 (3), pp. 155-156. https://doi. org/10.1089/bio.2010.7302

López-Briones, C., Amorós, R., Benkovic, V. y Zurriaga, O. (2014). Inventario de registros españoles de pacientes para la creación de un registro europeo de registros. Gaceta Sanitaria, 28 (Supl. C), p. 87 .

Martínez-Frías, M. L. (1995). Manual Operacional del ECEMC. Madrid: Martínez-Frías y Bermejo.

Montserrat Moliner, A. y Waligora, J. (2013). The European Union policy in the field of rare diseases. Public Health Genomics, 16 (6), pp. 268-277.

Morente, M. M., Álava, E. de y Fernández, P. L. (2007). Tumour Banking: The Spanish Design. Pathobiology, 74, pp. 245-250. https://doi org/10.1159/000104452

Morente, M. M., Fernández, P. L. y Álava, E. de (2008). Biobanking: old activity or young discipline? Seminars in Diagnostic Pathology, 25 (4), pp. 317-322. https://doi.org/10.1053/j. semdp.2008.07.007

Norlin, L., Fransson, M. N., Eriksson, M., Merino-Martínez, R., Anderberg, M., Kurtovic, S. y Litton J. E. (2012). A minimum data set for sharing biobank samples, information, and data: MIABIS. Biopreservation and Biobanking, 10 (4), pp. 343-348. https://doi. org/10.1089/bio.2012.0003

Phillips, W. R. (2004). Zebras on the commons: rare conditions in family practice. The Journal of the American Board of Family Practice, 17 (4), pp. 283-286. https://doi.org/10.3122/jabfm.17.4.283

Porta, M. (ed.). (2014). A dictionaryofepidemiology (6. a ed.). New York: Oxford University Press. https://doi.org/10.1093/ acref/9780199976720.001.0001

Rath, A., Olry, A., Dhombres, F., Brandt, M. M., Urbero, B. y Ayme, S. (2012). Representation of rare diseases in health information systems: The Orphanet approach to serve a wide range of end users. Human Mutation, 33 (5), pp. 803-808. https://doi. org/10.1002/humu.22078

Riegman, P. H., Dinjens, W. N. y Oosterhuis, J. W. (2007). Biobanking for interdisciplinary clinical research. Pathobiology, 74 (4), pp. 239-244. https://doi.org/10.1159/000104451

Riegman, P. H., Morente, M. M., Betsou, F., Blasio, P. de y Geary, P. (2008). Biobanking for better healthcare. Molecular Oncology, 2 (3), pp. 213222. https://doi.org/10.1016/j.molonc.2008.07.004

Rubinstein, Y. R., Groft, S. C., Bartek, R., Brown, K., Christensen, R. A., Collier, E. [...] y Vaught, J. (2010). Creating a global rare disease patient registry linked to a rare diseases biorepository database: Rare Disease-HUB (RD-HUB). Contemporary Clinical Trials, 31 (5), pp. 394-404. https:// doi.org/10.1016/j.cct.2010.06.007

Schieppati, A., Henter, J.-I., Daina, E. y Aperia, A. (2008). Why rare diseases are an important medical and social issue. The Lancet, 371 (9629), pp. 2039-2041. https://doi.org/10.1016/ s0140-6736(08)60872-7

Simeon-Dubach, D., Burt, A. D. y Hall, P. A. (2012). Quality really matters: the need to improve specimen quality in biomedical research. Histopathology, 61, pp. 1003-1005. https://doi. org/10.1111/his. 12060

Simeon-Dubach, D. y Watson, P. (2014). Biobanking 3.0: Evidence based and customer focused biobanking. Clinical Biochemistry, 47 (4-5), pp. 300308. https://doi.org/10.1016/j.clinbiochem.2013.12.018

Taruscio, D., Kodra, Y., Ferrari, G. y Vittozzi, L. (2014). The Italian National Rare Diseases Registry. Blood Transfusion, 12 (Supl. 3), pp. s606-613. 
Thompson, R., Johnston, L., Taruscio, D., Monaco, L., Béroud, C., Gut, I. G. [...] y Lochmüller, H. (2014). RD-Connect: An Integrated Platform Connecting Databases, Registries, Biobanks and Clinical Bioinformatics for Rare Disease Research. Journal of General Internal Medicine, 29 (Supl. 3), pp. 780-787. https://doi.org/10.1007/ s11606-014-2908-8

Veloza Cabrera, L. A., Wiesner Ceballos, C., Serrano López M. L., Peñaranda Correa, N. R. y Huertas Salgado A. (2010). Consideraciones éticas y legales de los biobancos para investigación. Revista Colombiana de Bioética, 5 (1), pp. 121-141. https://doi. org/10.18270/rcb.v5i1.1287

Zurriaga Lloréns Ó., Martínez García, C., Arizo Luque, V., Sánchez Pérez, M. J., Ramos Aceitero, J. M., García Blasco, M. J. [...] y Posada de la Paz, M. (2006). Los registros de enfermedades en la investigación epidemiológica de las enfermedades raras en España. Revista Española de Salud Pública, 80 (3), pp. 249-257. https://doi.org/10.1590/S113557272006000300005

\section{Legislación}

Council Recommendation of 8 June 2009 on an action in the field of rare diseases (2009/C 151/02). Official Journal of the European Union, 151, pp. 7-10. Disponible en: https://eur-lex.europa.eu/LexUriServ/LexUriServ.do?uri= OJ:C:2009:151:0007:0010:EN:PDF

Ley de Investigación Biomédica 14/2007, de 3 de julio. Boletín Oficial del Estado núm. 159, 4 julio 2007, pp. 2882628846. Disponible en: https://www. boe.es/boe/dias/2007/07/04/pdfs/ A28826-28848.pdf

Real Decreto 1716/2011, de 18 de noviembre. Boletín Oficial del Estado núm. 290, 2 diciembre 2011, pp.128434128454. Disponible en: https://www. boe.es/boe/dias/2011/12/02/pdfs/ BOE-A-2011-18919.pdf

\section{Informes y otros recursos en línea}

European Commission. Biobanking and Biomolecular Resources Research Infrastructure (BBMRI). [En línea]. Disponible en: https://cordis.europa.eu/ project/rcn/88365_en.html

European Commission. Biobanks for Europe. A challenge for governance. Report of the Expert Group on Dealing with Ethical and Regulatory Challenges of International Biobank Research, 2012. [En línea]. https:// doi.org/10.2777/68942

European Platform for Rare Disease Registries (EpiRare). Common Data Set and disease-, treatment and other specific modules. I-Report on the survey on Common Data Elements. [En línea]. Disponible en: http://www. epirare.eu/_down/del/D9.1_ReportontheSurveyonCDE_FINAL.pdf

European Union Committee of Experts on Rare Diseases (EUCERD). EUCERD Core Recommendations on Rare Disease Patient Registration and Data Collection, 2013. [En línea]. Disponible en: http://www.eucerd.eu/ wp-content/uploads/2013/06/EUCERD_Recommendations_RDRegistryDataCollection_adopted.pdf

European Union Committee of Experts on Rare Diseases (EUCERD). 2014 Report on the State of the Art of Rare Disease Activities in Europe, 2014. [En línea]. Disponible en: http://www.eucerd. eu/upload/file/Reports/2014ReportSt ateofArtRDActivities.pdf

International Rare Diseases Research Consortium (IRDiRC). Policies \& Guidelines. 2013. [En línea]. Disponible en: http://www.irdirc.org/ wp-content/uploads/2017/10/IRDiRC_ policies_24MayApr2013.pdf

JRC Scientific and Technical Reports. Biobanks in Europe: Prospects for Harmonisation and Networking, 2010. [En línea]. Disponible en: http://www.eurosfaire.prd.fr/7pc/doc/1280153287_ biobanks_eu_jrc57831.pdf
Medical Research Council. (2001). Human tissue and biological samples for use in research. Operational and ethical guidelines. [En línea]. Disponible en: http://www.who.int/genomics/elsi/regulatory_data/region/ euro/102/en/

National Organization for Rare Disorders (NORD), Rare Diseases Europe (EURORDIS) y Canadian Organization for Rare Diseases (CORD). EURORDISNORD-CORD Joint Declaration 10 Key Principles of Rare Disease Patient Registries. [En línea]. Disponible en: http://download.eurordis.org/documents/pdf/EURORDIS_NORD_CORD_ JointDec_Registries_FINAL.pdf

Organization for Economic Co-Operation and Development (OECD). OECD Best Practice Guidelines for Biological Resource Centres, 2007. [En línea]. Disponible en: http://www.oecd.org/sti/ emerging-tech/38777417.pdf

Orphanet Report Series. Rare Disease collection. Rare Disease Registries in Europe, 2014. [En línea]. Disponible en: http://www.orpha.net/orphacom/ cahiers/docs/GB/Registries.pdf

Rare Diseases Task Force. Patient registries in the field of rare diseases. Overview of the issues surrounding the establishment, management, governance and financing of academic registries, 2009/2011. [En línea]. Disponible en: http://www.eucerd. eu/?post_type=document $\& p=1218$

Red Nacional de Biobancos (RNB). Plan estratégico de la RNB. [En línea]. Disponible en: http://www.redbiobancos.es

U. S. Department of Health and Human Services. National Institutes of Health. National Cancer Institute. Office of Biorepositories and Biospecimen Research. $\mathrm{NCl}$ Best Practices for Specimen Resources. [En línea]. Disponible en: http://biospecimens. cancer.gov/bestpractices/2011-NClBestPractices.pdf 